\title{
Eine Krise der liberalen Fortschrittsidee? Anmerkungen zu Ivan Krastevs Krisendiagnose
}

\author{
Buchbesprechung zu: Ivan Krastev. Europadämmerung. \\ Ein Essay. Berlin: Suhrkamp, 2017
}

Sebastian M. Büttner (sebastian.buettner@uni-due.de)

University of Duisburg-Essen, Germany

In den letzten Jahren sind einige Bücher zu den diversen Krisen und zur Malaise der Europäischen Integration erschienen. Das Buch Europadämmerung des bulgarischen Politologen und Publizisten Ivan Krastev ist meiner Ansicht nach eines der herausragendsten. Der im Jahr 2017 bei Suhrkamp publizierte Essay (er war im gleichen Jahr unter dem Titel After Europe zunächst auf Englisch erschienen) wurde relativ bald nach Erscheinen vom Feuilleton ausführlich besprochen und gewürdigt. Auch hatte Krastev in den vergangenen Jahren einige Gastbeiträge in deutschsprachigen Zeitungen. Dennoch haben Krastevs pointiert vorgetragene Thesen, insbesondere seine provokante These, dass Europas Desintegration bereits voll im Gange ist, in der Europasoziologie bisher relativ wenig Widerhall gefunden.

Ein Grund für die noch geringe Resonanz von Krastevs Thesen in der aktuellen Europaforschung mag schlicht in der Eigenzeit wissenschaftlicher Publizistik liegen, die der Tagespolitik und tagesaktuellen Medienberichterstattung in der Regel weit hinterherhinkt. Es kann zudem auch daran liegen, dass Ivan Krastev kein klassischer Wissenschaftler ist, sondern ein unabhängiger politischer Kommentator, Publizist und Politikberater. Krastevs Biographie spiegelt jedenfalls keine klassische Universitätslaufbahn wider. Er bekleidet aktuell keine Professur, sondern er ist Vorsitzender des Think Tanks Centre for Liberal Strategies in Sofia und changiert als Permanent Fellow am Institut für die Wissenschaft vom Menschen gewissermaßen am Grenzbereich von Wissenschaft und Politikberatung. Dies sollte für die akademische Europaforschung jedoch kein Grund sein, sich nicht intensiver mit Krastevs Thesen auseinanderzusetzen.

Ein weiterer Grund für die geringe Rezeption von Krastevs Buch in der aktuellen akademischen Europa-Debatte mag auch in der Herkunft des Autors zu sehen sein. So eigenartig das klingen mag: Es lässt sich durchaus feststellen, dass die Wissenschaften - und nicht zuletzt auch die Europaforschung - von Selektivitäten und Hierarchien in der Wahrnehmung, Einschätzung und Anerkennung von Diskurspositionen geprägt sind. Westliche Positionen sind hier zumeist dominant, allen voran die Stimmen von Vertreter*innen nordeuropäischer und nordamerikanischer Spitzenuniversitäten. Beiträge aus Südeuropa und auch Beiträge von osteuropäischen Kolleg*innen zählen nur sehr selten zum Mainstream der Diskurse - und wenn doch, dann häufig nur vermittelt über die dominanten westeuropäischen und nordamerikanischen Institutionen und Sprecherpositionen. 
Ich würde diesen Aspekt an dieser Stelle nicht so deutlich betonen, wenn die Herkunft Krastevs und sein dadurch geprägter Blickwinkel nicht ganz entscheidend wären für die besondere Perspektive des Autors. Es ist meines Erachtens eine zentrale Stärke des Buches, dass hier ein Zeitzeuge des Zusammenbruchs der Sowjetunion und ein erfahrener Chronist der Transformationen in Mittel- und Osteuropa über die gegenwärtige Situation Europas, vor allem der Europäischen Union, schreibt und dabei auch ganz explizit an seine Erfahrungen des Umbruchs um das Jahr 1989 anknüpft. Krastev betont zum einen, dass ihm seine Erfahrung gelehrt habe, dass tiefgreifende gesellschaftliche Umbrüche in der Regel nicht disruptiv, etwa per Revolution oder von heute auf morgen, vonstattengingen. Sie vollzögen sich häufig eher leise und unbemerkt, nicht selten als nicht-intendierte Folge von geringfügigen politischen Verschiebungen und gesellschaftlichen Entwicklungen. Des Weiteren bringt Krastev in seiner Analyse eine gewisse Distanz zum liberalen Fortschrittsnarrativ zu Ausdruck, das die Politik, die Sozialwissenschaften und in großen Teilen auch die Gesellschaften des Westens nach dem Ende des Kalten Krieges nachhaltig gekennzeichnet habe. Paradigmatisch verkörpert sieht Krastev dieses Narrativ vor allem in Francis Fukuyamas geflügelter These vom „Ende der Geschichte“ aus dem Jahr 1989. Wie falsch und auch irreführend sich die Annahmen von Fukayama gerade aus heutiger Perspektive erwiesen haben, dies diskutiert Krastev ausführlich im ersten Teil seines Buches (insbesondere auf den Seiten 25-36). Er stellt Fukuyama die Thesen des Politikwissenschaftlers Ken Jowitt entgegen, der in den Debatten um Fukuyamas Thesen bereits im Jahr 1991 mit seiner Deutung einer "Neuen Weltunordnung" kritisch auf Fukuyama Bezug genommen hatte (Jowitt, 1991, 1992). Dieser Ansatz von Jowitt bildet den Grundton von Krastevs Europadämmerung.

In der unterschiedlichen Erfahrung der Systemtransformation von 1989 macht Krastev schließlich noch einen bedeutenden Mentalitätsunterschied zwischen West- und Osteuropa aus, der für die Einschätzung der heutigen Krise der Europäischen Integration nicht unerheblich ist. Während, so Krastev, viele Leute in Westeuropa aufgrund der Erfahrung des Siegeszugs von Demokratie und Kapitalismus weiterhin glauben, „alles werde schon gut werden" (19), seien vielen Menschen in Osteuropa, die bereits die Erfahrung eines umfassenden Systemwandels am eigenen Leibe mitgemacht haben, häufig weitaus skeptischer und auch ängstlicher in der Einschätzung aktueller politischer und gesellschaftlicher Entwicklungen. Es ist genau diese Skepsis, die die fatalistisch anmutende These einer europäischen „Desintegration“ prägt. Dies bringt der Autor in der Einleitung seines Buches unmissverständlich zum Ausdruck: „Ich gehöre zu denen, die glauben, dass der Desintegrationszug den Brüsseler Hauptbahnhof bereits verlassen hat - und ich befürchte, dass dies den Kontinent in Unordnung stürzen wird (...)“ (16f.).

Nun ließe sich einwenden, dass Krastev einen Graben zwischen Ost- und Westeuropa betont und damit reifziert, der so und in dieser Form heute überhaupt nicht mehr existiert. Des Weiteren ließe sich Krastevs Buch mit dem Verweis auf die Probleme und Fallstricke von allzu alarmistisch vorgetragenen Thesen und Behauptungen relativ einfach vom Tisch wischen. Als reflektierter Sozialwissenschaftler, so könnte man Krastev entgegenhalten, müsse man stets im Auge behalten, dass man durch die Analyse und Beschreibung von gesellschaftlichen und politischen Ereignissen immer auch an der Konstruktion und Deutung der gesellschaftlichen Wirklichkeit mitwirkt - dass es also keine neutralen Beschreibungen gibt und dass es auch in der Verantwortung des Autors liegt, mit zu reflektieren, welche gesellschaftlichen Implikationen die eigenen Analysen und Begriffe mit sich bringen. 
Man könnte dem Autor sicher all dies vorwerfen, und man könnte an vielen Stellen sicher auch mehr empirische Evidenz, mehr begriffliche Schärfe und eine stärkere inhaltliche Differenzierung einfordern. Es wäre meiner Ansicht nach jedoch völlig verfehlt, den 140 Seiten langen „Essay“ von Krastev leichtfertig abzutun. Allein schon deshalb, weil der Autor seinen Standpunkt von Beginn an explizit deutlich macht, und weil er auch betont, dass es ihm keineswegs um eine normativ geleitete Abrechnung mit dem europäischen Einigungsprozess geht: „Dieses Buch will die EU weder retten noch betrauern. Es ist kein weiteres Traktat über die Ätiologie der europäischen Krise (...). Und erst recht ist es kein Buch eines Europaskeptikers" (18). Ich sehe gerade auch in der Betonung von strukturellen Unterschieden zwischen West- und Osteuropa - und vor allem auch in der Betonung der unterschiedlichen Wahrnehmung des Systemumbruchs 1989 - die besondere Stärke des Buches. In einer Zeit, in der die Besonderheiten der Gesellschaften und der Systemtransformation in Osteuropa in den Europastudien zunehmend in den Hintergrund gerückt sind und immer mehr zu einer Frage des Compliance und Nicht-Compliance mit europäischen Regeln und Standards stilisiert werden (s. auch den Beitrag von Worschech in diesem Heft), ist ein Verständnis für die strukturellen gesellschaftlichen Unterschiede zwischen Ost- und Westeuropa unabdingbar. Denn die neuen reaktionären und nationalistischen Bewegungen in den mittel- und osteuropäischen Mitgliedsländern lassen sich nicht nur als „Ausnahme" oder als "Abweichung" vom liberal-demokratischen Konsensus interpretieren. Spätestens mit der Ausbreitung neuer reaktionärer, betont anti-liberaler und anti-europäischer Bewegungen in Westeuropa sollte klar sein, dass es sich hier keineswegs um einen Sonderfall oder um eine Ausnahme handelt. Ein Verständnis der besonderen Erfahrungen und der besonderen gesellschaftlichen Konstellationen in Osteuropa kann somit dabei helfen, die allgemeine Krise Europas und die wachsende Skepsis der Europäer gegenüber der heutigen Europäischen Union besser zu verstehen.

Zugegeben: Krastev trägt seine Thesen pointiert, zuweilen auch zugespitzt, vor. Sein Ziel ist es jedoch nicht zu polemisieren, sondern die routinierte Europapolitik und die Europawissenschaften wachzurütteln und herauszufordern, neu und anders über den Prozess der europäischen Integration nachzudenken und sich dabei auch über die eigenen axiomatischen Beschränkungen und Perspektivenverengungen bewusst zu werden. Entgegen der Deutungen, die die aktuelle Krise der europäischen Integration lediglich als eine Krise der „institutionellen Architektur“ der Europäischen Union (etwa im Hinblick auf eine Korrektur der Währungsunion) oder als Folge des chronischen Demokratiedefizits der Europapolitik interpretieren, formuliert Krastev die These eines viel grundlegenderen Wandels des bisherigen Modus europäischer Integration und der etablierten Formen demokratischer Politik, der sich spätestens seit der Zuspitzung der Flüchtlingskrise immer deutlicher abzeichne: Im „Zeitalter der Migration“, so Krastev, werde die Demokratie zunehmend zu einem Instrument des Ausschlusses statt der Inklusion (22). Während die westlichen liberalen Demokratien bis vor kurzem darauf ausgerichtet waren, vor allem „bedrohte Minderheiten“ zu schützen und zu integrieren, hätten sich nun „bedrohte Mehrheiten“ zu einer bedeutenden politischen Kraft aufgeschwungen, um gegen die etablierte Politik zu rebellieren. Es handle sich hier jedoch nicht bloß um einen „populistischen Aufstand gegen das Establishment“, so Krastev (21), sondern um eine „Rebellion der Wähler gegen die meritokratischen Eliten“. Krastev sieht hier vor allem die Flüchtlingskrise und nicht etwa die Währungskrise, das Brexit-Referendum oder den Rechtsruck als entscheidendes Ereignis. Die Flüchtlingskrise sei, so Krastev, „Europas 11. September“ (25). 
Dies klingt zunächst nach einem recht eindimensionalen monokausalen Erklärungsansatz. Krastevs Argument ist jedoch keineswegs monokausal gemeint. Er sieht in der Zuspitzung der Flüchtlingskrise in den vergangenen Jahren lediglich einen gesellschaftlichen Umschlagpunkt, an dem gesellschaftliche Gruppen und politische Einstellungen zunehmend in den Mittelpunkt rücken, die vorher stark in der Defensive, wenn nicht gar marginalisiert worden waren. Krastev zielt in seinem Buch folglich darauf ab, Antworten auf die Fragen zu geben wie die Flüchtlingskrise Europa verändert (hat) und warum die Bürger die etablierten politischen Kräfte und insbesondere auch die „Brüsseler Bürokratie“ zunehmend verachten (21).

Wie oben bereits angedeutet, sieht Krastev hier vor allem ein Versagen des Liberalismus beziehungsweise ein Versagen der liberalen Fortschrittserzählung, deren Widersprüche und utopische Versprechen im Zuge der Flüchtlingskrise für viele Menschen offen zutage getreten seien. Es sei das „Versagen des Liberalismus bei der Bewältigung des Migrationsproblems" und nicht die Wirtschaftskrise oder die wachsende soziale Ungleichheit, das für Krastev zentral erklärt, „warum die Öffentlichkeit sich gegen inn [den Liberalismus] wendete" (32). Die Unfähigkeit und die mangelnde Bereitschaft liberaler Eliten, die Migration und deren Folgen zum Gegenstand der Diskussion und der politischen Auseinandersetzung zu machen, wie auch die Behauptung, die gegenwärtige Politik sei für alle Beteiligten von Vorteil („eine Win-Win-Situation“), habe dazu geführt, dass der Liberalismus in den Augen vieler zum "Synonym von Heuchelei“ geworden sei. Diese „Revolte gegen die Heuchelei der liberalen Eliten" habe die politische Landschaft Europas radikal verändert.

Krastev bewegt sich mit seiner Argumentation und mit der Zuspitzung seiner These auf die „Migrationsproblematik" und auf eine Kritik „liberaler Eliten“ und des vermeintlichen „liberalen Common Sense" durchaus auf einem sehr schmalen Grat. Sind es nicht gerade die Agitator*innen der neuen Rechten, die ebenjenes Narrativ von der "Migrationskrise“ als „Mutter aller Krisen“ und von der Schwäche (oder gar vom Schaden!) des Liberalismus für den Fortbestand der "Gesellschaft" (hier verstanden als nationale ethnische Gemeinschaften) bedienen? Darüber hinaus ist kritisch zu fragen, ob sich die Diskussion über die Flüchtlingskrise, wie Krastev behauptet, tatsächlich so deutlich von wirtschaftlichen Fragen und von der Zuspitzung der sozialen Frage beziehungsweise von der Diskussion über Zunahme sozialer Ungleichheiten trennen lässt? Ich glaube jedenfalls, dass es sich nicht trennen lässt und dass in der Ablehnung „der Eliten“ und vor allem auch der „Brüsseler Eliten“ ein Ungleichheitskonflikt zutage tritt - ein allgemeiner symbolischer Konflikt um Anerkennung, aber auch ein ganz manifester Konflikt um politische Partizipation und Repräsentation, der umso stärker wird je mehr Bevölkerungsgruppen das Gefühl haben, dass „die Politik“ und die offiziellen staatlichen Institutionen ihre Bedürfnisse und Interessen zu wenig berücksichtigen. Je mehr dieser Eindruck durch offensichtliche Fehler und durch Fehlverhalten der etablierten Politik und je mehr der Konflikt durch entsprechende politische Akteure und Bewegungen verstärkt wird, desto mehr spitzt sich die Legitimitätskrise der liberalen Demokratie zu (vgl. Büttner, 2018).

Wenn man Krastevs Argumentation jedoch vor allem hermeneutisch und nicht wertend versteht - also ganz klassisch als ein Bemühen um ein tieferes Verstehen der Zusammenhänge und der Sinndeutungen der Akteure - liest man seine Analyse der aktuellen politischen Krise mit viel Gewinn. Krastev bietet nämlich viele konkrete Anhaltspunkte zum Verständnis des derzeitigen Rechtsrucks in Europa, und er weist auf die vielen Widersprüche der aktuellen politischen Lage in Europa hin, die eine klare und eindeutige Interpretation 
der Entwicklung Europas so erschweren: etwa der Umstand, dass gerade in den vermeintlich „gefestigten Demokratien“ eine wachsende Zahl der Bevölkerung immer skeptischer wird gegenüber ihren politischen Eliten und immer zynischer gegenüber der Demokratie (75); oder die Frage, warum nachweislich korrupte oder charakterlich fragwürdige Demagogen heute einen immer größeren Zulauf erhalten und die etablierten, überwiegend meritokratisch und sachlich orientierten politischen Eliten misstrauisch beäugt, wenn nicht gar rigoros abgelehnt werden.

Krastev vermittelt hier - gerade mit Blick auf die rechtspopulistische und rechtskonservative Wende in den neuen Mitgliedsländern in Mittel- und Osteuropa - eine Reihe von interessanten Einsichten. Er verweist zum einen auf Parallelen zur ersten Phase der Dekolonialisierung in den 1960er Jahren. Ähnlich wie die französischen pieds noirs, die während des Unabhängigkeitskrieges gezwungen waren, Algerien zu verlassen, repräsentiere der Rechtspopulismus der Gegenwart nicht die „Sehnsüchte der Unterdrückten“, sondern die „Frustration der Mächtigen“. Der heutige Rechtspopulismus sei kein Populismus „des Volkes", der wie vor einem Jahrhundert in einem romantischen Nationalismus gefangen ist. „Er basiert vielmehr auf demographischen Prognosen, die Europa eine sinkende Bedeutung in der Welt prophezeien und auf der Erwartung eines massenhaften Zustroms von Menschen nach Europa" (34f.).

Krastev betont zweitens unter Verweis auf aktuellere sozialpsychologische AutoritarismusStudien der Sozialpsychologin Karen Stenner (2005), dass bestimmte gesellschaftliche Situationen, die als bedrohlich wahrgenommen werden oder eine politische Rhetorik, die Bedrohungen heraufbeschwört, den Wunsch nach einem rigorosen Durchgreifen und nach einer autoritären Führung befördern können (49ff.). Nicht alle Bedrohungen beschwören nach Stenner jedoch diese Reaktion herauf, sondern vor allem solche, die als eine „normative Bedrohung" wahrgenommen werden. Dies seien vor allem jene Situationen, die das Gefühl auslösen, dass die gesamte moralische Ordnung in Gefahr sei. Auf dieser Basis interpretiert Krastev den besonderen Erfolg - und nicht zuletzt auch die besondere moralische Rechtfertigung - von Tabubrechern wie Trump oder der Demagogen der Leave-Kampagne in Großbritannien. Dieser Erfolg lasse sich, so Krastev, vor allem durch ihre Fähigkeit erklären, die Wähler davon zu überzeugen, dass eine gewisse Grenze überschritten sei. Ein ähnliches Muster erkennt man heute wohl auch in den Debatten um einen „Kontrollverlust“ ausgelöst durch eine „massenhafte Zuwanderung" von Flüchtlingen und anderen Migrantengruppen.

Mit Blick auf die besonders starke Ablehnung gegenüber Migration in allen postsozialistischen Ländern geht Krastev drittens ausführlich auf die Spezifika des Rechtsrucks in Mittelund Osteuropa ein (55ff.). Besonders paradox erscheint hier die mehrheitliche Ablehnung von Migration und Liberalismus gerade im Hinblick auf die EU-Mitgliedsländer in Mittelund Osteuropa, sehnten sich die Bevölkerungen dort doch einst in besonderer Weise, so meint man, nach einer „Rückkehr nach Europa“. Auch gehören Menschen aus Mittel- und Osteuropa zu den mobilsten in Europa, und sie profitieren seit Jahren von offenen Grenzen, Arbeitsmigration und vom Zugang zu europäischen Arbeitsmärkten. Bei genauerem Hinsehen finde man, so Krastev, jedoch eine ganze Reihe von Faktoren, die die gesellschaftliche Situation und die stark ausgeprägte Ablehnung kosmopolitischer Werte in Mittel- und Osteuropa zu erklären vermögen (58ff.). Er weist hier vor allem auf spezifische geschichtliche Faktoren (etwa auf die verspätete Nationalstaatsgründung im Vergleich zu Westeuropa, aber die Erfahrung der Fremdherrschaft zu Zeiten der Sowjetunion), auf problematische demographische Entwicklungen (etwa auf massenhafte Abwanderung und die Angst vor dem „Aussterben“ ganzer Landstriche oder der eigenen Bevölkerung) und 
auf Paradoxien der postkommunistischen Transformation (etwa auf das ausgeprägte Misstrauen gegenüber den Reformeliten und staatlichen Akteuren). Krastev nennt hier eine ganze Reihe von Gründen und Erklärungsfaktoren, die sich hier nicht alle widergeben lassen. Er zeichnet allerdings ein sehr eindrückliches gesellschaftliches Panorama der aktuellen gesellschaftlichen und politischen Situation in Mittel- und Osteuropa. Besonders lesenswert sind seine Erläuterungen zur spezifischen Form des postkommunistischen Nationalismus und Anti-Liberalismus: „In der Politik bedrohter Mehrheiten ist demokratisches Denken ein demographisches Denken. Die Nation ist - ähnlich wie Gott - einer der Schutzschilde der Menschen gegen den Gedanken der Sterblichkeit" (62). So könne es nicht überraschen, so Krastev weiter, dass demographische Vorstellungen nicht nur die feindselige Haltung gegenüber Ausländern, sondern auch deren negative Reaktionen auf soziale Veränderungen wie die Homo-Ehe bestimmten. Für viele Konservative bedeute die Homo-Ehe weniger Kinder und damit einen weiteren Bevölkerungsrückgang. Mit diesem Verweis auf demographische Fragen und auf die Angst vor einem „ethnischen Verschwinden“ (61) bringt Krastev ein Motiv auf den Punkt, das auch in Deutschland und vielen anderen Ländern in der Migrationsdebatte durch Formeln wie „Deutschland schafft sich ab“ (Sarazin, 2010) oder „Der große Austausch“ (Camus, 2016) immer wieder ins Feld geführt wird.

Krastev geht im zweiten Teil seines Essays mit dem Titel „Sie, das Volk“ (73-126) schließlich noch ausführlicher auf das "Gespenst des Populismus“ (76ff.) im heutigen Europa ein und diskutiert insbesondere die Frage, warum sich die politische Landschaft in Europa derzeit so polarisiert hat und dabei vor allem die "Kosmopoliten" und die "meritokratischen Eliten“ so stark in die Kritik geraten sind. Er verweist hier einerseits auf ein „Paradoxon der liberalen Demokratie“, wonach sich die Menschen immer machtloser fühlten, je freier sie seien (88f.). Dieses Gefühl der Machtlosigkeit erzeuge eine neue Sehnsucht nach Eindeutigkeit und Klarheit bei politisch umkämpften Fragen. Der Erfolg des Populismus gründet, so Krastev, vor allem auf der Strategie, dass er ein Gefühl der Geborgenheit und Identität vermittelt, das die liberale Demokratie und erst recht nicht die kühle rational-legale Herrschaft der Europäischen Union zu vermitteln vermögen. Hier sieht Krastev auch den zentralen Grund der Ablehnung der etablierten Parteien („das Establishment“) und der meritokratischen Eliten in Brüssel („die Eurokraten“): Diese werden, so Krastev, von den „bedrohten Mehrheiten“ stets misstrauisch beäugt, weil sie nicht als „Loyalitätseliten“ (105) und damit als die „Unsrigen“, wahrgenommen würden, sondern im Zweifelsfall verschwinden oder fremden Interessen dienten.

Genau darauf gründet sich der Erfolg der heutigen reaktionären und anti-liberalen Bewegungen. In den Augen Ivan Krastevs bildet dieser „Kampf um den Charakter und die Pflichten der Eliten“ geradezu den „Kern“ der gegenwärtigen populistischen Herausforderung. Doch anders als noch vor einem Jahrhundert interessierten sich die heutigen Anführer nicht mehr für Verstaatlichung. „Sie versprechen den Menschen nicht, sie zu retten, sondern bei ihnen zu bleiben. Sie versprechen, die nationalen und ideologischen Beschränkungen wiederherzustellen, die von der Globalisierung aufgehoben wurden. Sie loben das Volk, weil es keine Fremdsprachen beherrscht und nirgendwo anders hingehen kann. Kurz gesagt, Populisten versprechen ihren Wählern nicht Kompetenz, sondern Intimität. Sie versprechen, das Band zwischen Eliten und Volk wiederherzustellen. Und eine rasch wachsende Zahl von Menschen in Europa findet dieses Versprechen heute attraktiv" (108). 
Diese letzte zitierte Passage zeigt nochmals sehr eindrücklich, in welchem Stil und in welchem Tonfall der Text von Krastev geschrieben ist. Sie verdeutlicht vor allem aber auch sehr gut Krastevs These von der Dialektik der liberalen Demokratie und der kosmopolitischen Vision Europas. Der Autor vermittelt mit seinen Analysen zu den geistigen, sozialpsychologischen und soziologischen Grundlagen des heutigen Populismus zweifelsohne sehr interessante und erhellende Einsichten für ein besseres Verständnis der gesellschaftlichen Situation und der politischen Krise Europas. Die Frage ist nun: Was folgt aus diesen Analysen?

Krastev selbst bleibt hier relativ vage und unbestimmt. Er diskutiert - wohl damals noch stark vom Ausgang des Brexit-Referendums im Juni 2016 beeindruckt - einige problematische Aspekte von Volksentscheiden und skizziert die Fallstricke direkter Bürgerbeteiligung. Er zeigt, dass Volksentscheide von Regierungen und politischen Parteien auf sehr unterschiedliche Art und Weise strategisch eingesetzt und genutzt werden und skizziert hierbei paradigmatisch drei unterschiedliche Optionen: 1) eine „mutige“ Variante: das Verfassungsreferendum von Matteo Renzi im Dezember 2016, 2) eine "niederträchtige“ Variante": die Abstimmung über die Ratifizierung eines Assoziierungsabkommens zwischen der EU und der Ukraine in den Niederlanden im April 2016, und 3) eine "hässliche“ Variante: Ungarns Referendum über die Frage der Aufnahme von Flüchtlingen im Rahmen der EU-Asylpolitik vom Oktober 2016. Alle drei Referenden sind für Krastev Beispiele dafür, wie in Europa derzeit auf nationaler Ebene ein ausgeprägter Europapessimismus gefördert wird, „der weit über die Europaskepsis der letzten Jahre hinausgeht“ (125). Im Schlusskapitel diskutiert Krastev unter dem Schlagwort „Überlegungen zur Zerbrechlichkeit und Widerstandsfähigkeit Europas" aktuelle Wege Europas durch die Krise. Er mahnt hier schließlich einerseits zu „Improvisations-“ und zu „Kompromissfähigkeit“. Mehr als diese allgemeinen Ratschläge zum Umgang mit der Krise und mögliche Wege aus der Krise enthält der Schluss jedoch nicht. Dies zeigt, dass es dem Politikberater Ivan Krastev tatsächlich nicht darum ging, eine gut umsetzbare Anleitung zur Bewältigung der Krise Europas zu liefern, sondern in erster Linie um Analyse und um ein Verständnis der aktuellen Situation.

Über die politischen Implikationen seiner Analyse muss und sollte intensiv diskutiert und gestritten werden. Von den Europawissenschaften, insbesondere von der Europasoziologie, erhoffe ich mir, dass sie sich intensiv mit Krastevs Thesen und mit seinen Interpretationsangeboten auseinandersetzt. Er hat mit seiner provokanten These einer Zuspitzung der politischen Krise in Europa und mit seinem Verweis auf die vielen stillen Revolutionen und Systemzusammenbrüche Anstöße geliefert für eine intensivere Auseinandersetzung mit der Stagnation, wenn nicht gar der Desintegration, des europäischen Einigungsprojekts. Mit dem Hinweis auf die Widersprüche und Probleme der liberalen Fortschrittsideen stellt er viele Grundannahmen und Glaubenssätze der politischen Praxis und der politischen Wissenschaften in Frage. Die Analyse zeigt jedoch nicht nur die Widersprüche des liberalen Denkens auf, sondern ebenso die soziologischen Bedingungen und die geistigen und ideologischen Beschränkungen aktueller reaktionärer Bewegungen.

\section{Literatur}

Büttner, S. M. (2018). Eurokratiekritik - Ausdruck eines Ungleichheitskonflikts? Zur Veränderung demokratischen Regierens im heutigen Europa. In M. Eigmüller \& N. Tietze (Hrsg.), Ungleichheitskonflikte in Europa: Jenseits von Klasse und Nation (151-169). Berlin: Springer VS. 
Camus, R. (2016). Revolte gegen den Großen Austausch. Schnellroda: Antaios.

Jowitt, K. (1991). After Leninism: The new world disorder. Journal of democracy 2, 11-20.

Jowitt, K. (1992). The new world disorder: The Leninist extinction. Berkeley: University of California Press.

Sarrazin, T. (2010). Deutschland schafft sich ab. Wie wir unser Land aufs Spiel setzen. München: Deutsche Verlags-Anstalt.

Stenner, K. (2005). The authoritarian dynamic. Cambridge: Cambridge University Press. 